\title{
Uso de Hiperdatos en un Laboratorio de Electrónica (Códigos QR) Use of Hyperdata in a Laboratory of Electronics (QR Codes)
}

\author{
Carlos Sánchez-Azqueta ${ }^{1}$, Cecilia Gimeno ${ }^{2}$, Santiago Celma ${ }^{1}$, Esther Cascarosa ${ }^{3}$, Concepción Aldea ${ }^{1}$ \\ csanaz@unizar.es, cecilia.gimenogasca@uclouvain.be, \{scelma, ecascano,caldea\}@unizar.es
}

\author{
${ }^{1}$ Departamento de Ingeniería Electrónica y \\ Comunicaciones \\ Universidad de Zaragoza \\ Zaragoza, España
}

\author{
${ }^{2}$ ICTEAM Institute - ECS Group \\ Université Catholique de Louvain \\ Louvain-la-Neuve, Bélgica
}

\author{
${ }^{3}$ Departamento de Didáctica de Ciencias \\ Experimentales \\ Universidad de Zaragoza \\ Zaragoza, España
}

\begin{abstract}
Resumen- Las tecnologías de información y la comunicación (TICs) son un instrumento privilegiado para promover y facilitar el aprendizaje significativo de los alumnos. El uso de recursos multimedia y entornos virtuales de aprendizaje ya ha sido adoptado en numerosas instituciones de educación superior como parte de un proceso de aprendizaje mixto. Otra herramienta disponible para la innovación pedagógica es la realidad aumentada (RA), que permite la combinación de la información digital y física en tiempo real usando diversos dispositivos tecnológicos. De entre las realizaciones de realidad aumentada podemos distinguir diversos niveles, que comienzan con los códigos en 2D (códigos QR). Este trabajo presenta la generación de una serie de recursos multimedia, accesibles mediante códigos QR, para las sesiones prácticas en un laboratorio de Electrónica. Con el uso de dichos recursos se ofrece información contextualizada adicional, accesible por los alumnos cuando lo necesiten, lo que mejora el proceso de aprendizaje en el laboratorio.
\end{abstract}

Palabras clave: códigos QR, aprendizaje mediante TICs, mlearning, aprendizaje ubicuo

Abstract- Information and communication technologies (ICTs) are a privileged instrument to promote and facilitate meaningful student learning. The use of multi-media resources and virtual learning environments has already been adopted in many higher education institutions as part of a mixed learning process. Another tool available for teaching innovation is augmented reality (AR), which allows a combination of digital and physical information in real time using different technological devices. Within the augmented reality, we can find several levels, which start with the 2D codes (QR codes). This work proposes the generation of a set of multi-media resources, accessible by means of QR codes, for the practical sessions in a laboratory of Electronics. With these resources, additional and contextual information is offered, which can be accessed when the student requires it, thus improving the experimental learning process.

Keywords: QR code, ICT-based learning, m-learning, ubiquitous learning

\section{INTRODUCCIÓN}

Las tecnologías de la información y la comunicación (TICs) se han convertido en un elemento clave en la evolución de las estrategias en educación, como se desprende de su implantación masiva en todos los niveles educativos. Una de las aplicaciones más utilizadas de TICs en educación es la generación de recursos multimedia tales como vídeos, presentaciones comentadas o infografías (Sánchez-Azqueta, 2015-1).
A tal fin contribuyen esencialmente dos aspectos. Por un lado, la proliferación de aplicaciones móviles gratuitas que facilitan la tarea de creación y edición de dichos contenidos, así como su consumo ubicuo en lo que se conoce como $\mathrm{m}$ learning (Cheon, 2012; Motiwalla, 2007; Pu, 2011); por otro lado, el esfuerzo que llevan a cabo las instituciones educativas en la puesta en marcha, mantenimiento y mejora de plataformas digitales en las que alojar dichos contenidos.

Una de las cuestiones más críticas del uso de recursos multimedia en educación, y particularmente en educación superior, es cómo conseguir facilitar el acceso a dichos contenidos en el momento en que estos sean relevantes. En ese sentido, elementos de carácter intrínsecamente digital como apuntes enriquecidos, e-books o presentaciones multimedia permiten de manera inmediata enlazar a los contenidos multimedia mediante hipervínculos situados a lo largo de su desarrollo (Sánchez-Azqueta, 2016). Sin embargo, todavía existen muchos materiales educativos que no están en formato digital y que, por tanto, no admiten la inclusión de hipervínculos. Éste es el caso, por ejemplo, de los manuales y bibliografía de referencia de las asignaturas, que tradicionalmente han recurrido a la inclusión de dichos materiales en soportes alternativos como DVDs o páginas web diseñadas a tal efecto como repositorio.

Un ámbito docente en el que la necesidad de introducir material multimedia complementario es especialmente destacable es el de las sesiones prácticas de laboratorio en disciplinas experimentales. En las sesiones de laboratorio, los estudiantes deben utilizar instrumentación compleja, cuyo manejo está recogido en los manuales de uso, así como reproducir técnicas de medida de nivel avanzado. A pesar de que ambos contenidos pueden trasladarse al ámbito multimedia con facilidad (como un documento de texto en el primer caso y como un vídeo o infografía en el segundo), conseguir que los alumnos tengan un acceso sencillo e inmediato a ellos durante la sesión práctica proporcionaría una mejora en el proceso de aprendizaje.

En este contexto cobran especial relevancia las aplicaciones de realidad aumentada (RA), ya que tienen la particularidad de permitir la combinación de información digital y física en tiempo real usando dispositivos móviles. La RA, por tanto, presenta muchas ventajas en el campo de la educación, ya que permite ofrecer información de manera contextualizada, presentándola además en el lugar y en el momento en que los 


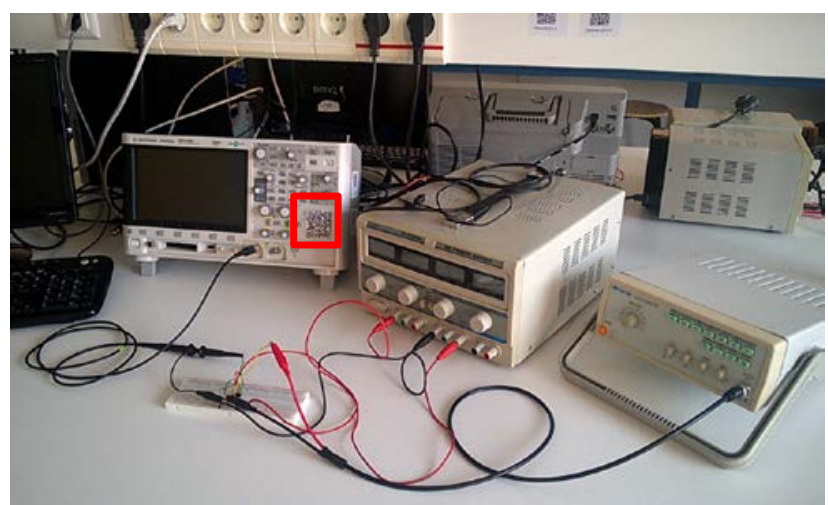

(a)

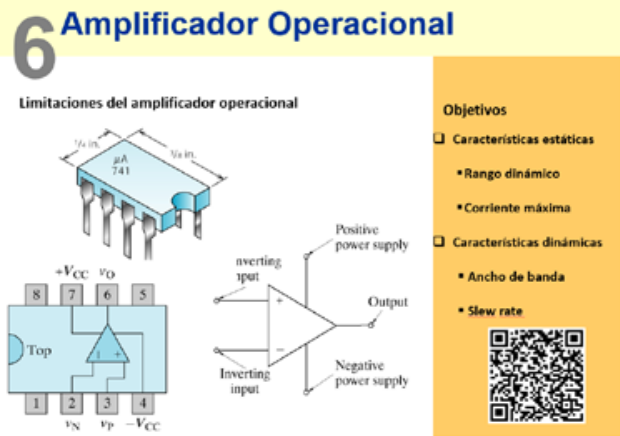

(b)

Fig 1. (a) Imagen de un puesto de laboratorio para realizar la caracterización del amplificador operacional, con el código QR a disposición del alumno y (b) detalle de la presentación de la práctica, con su correspondiente código QR

estudiantes la requieren, lo que ayuda a un enriquecimiento significativo del proceso de aprendizaje (Estebanell, 2012).

La incorporación de las TICs al ámbito educativo ha permitido el uso de nuevos recursos tales como la realidad aumentada con el objetivo de mejorar los procesos de enseñanza-aprendizaje. Las ventajas de los códigos QR en este sentido son su facilidad de uso y la enorme cantidad de información que pueden almacenar. Esto los convierte en recursos de gran potencial para la educación (Law, 2010; Kossey, 2015), aunque no todos los autores los clasifican como una categoría dentro de la realidad aumentada (Estebanell, 2012; Prendes, 2015; Cabero, 2016).

En este trabajo se presenta el desarrollo de una serie de recursos educativos complementarios para la realización de las sesiones prácticas en un laboratorio de Electrónica, y cómo se ha facilitado un acceso sencillo a ellos por parte de los alumnos mediante el uso de códigos QR.

\section{CONTEXTO}

Uno de los campos en los que se hace mayor hincapié en los planes de estudio de las disciplinas científicas y tecnológicas es la adquisición de competencias en el ámbito de la experimentación y del trabajo en el laboratorio. De esta manera, prácticamente desde el principio de sus estudios, los alumnos se enfrentan a sesiones prácticas en las que deben aplicar los conocimientos teóricos adquiridos en las distintas asignaturas a situaciones reales.

Además de competencias propias de cada titulación, como la operación con instrumentación específica, el manejo de hojas de características de componentes o la familiarización con las distintas medidas de seguridad que se aplican, los estudiantes adquieren competencias transversales como el trabajo en equipo, la redacción de informes de carácter técnico e, incluso, el trabajo por objetivos y por plazos, ya que las prácticas tienen una duración limitada en el tiempo. Todos ellos son altamente valorables en el ámbito profesional, y, al igual que sucede en un entorno profesional, se espera de los alumnos que sean capaces de resolver las cuestiones planteadas haciendo uso de sus conocimientos pero también de los recursos a su alcance (Sánchez-Azqueta, 2015-2).

Tradicionalmente, los alumnos han recibido una explicación teórico/práctica sobre los objetivos y procedimientos de cada sesión práctica previamente a su realización, y han contado con la ayuda de los profesores y el acceso al material específico de apoyo (manuales de instrucciones de la instrumentación, hojas de especificaciones de los componentes, etc.) durante el transcurso de cada sesión. Esta dinámica compromete en bastantes casos la posibilidad de dar respuesta inmediata y completa a las cuestiones y dudas planteadas por los alumnos, ya que los profesores deben atender a varios grupos simultáneamente durante cada sesión. Por otro lado, debido a que en muchas ocasiones los alumnos se encuentran con cuestiones similares, no se promueve un uso eficaz del tiempo de laboratorio.

Una solución que está ganando popularidad para mejorar este problema es la edición por parte del profesorado de cada asignatura de contenidos multimedia en los que se desarrollan los aspectos más relevantes de cada sesión práctica. De esta manera, mediante presentaciones comentadas, infografías o incluso vídeos, se puede ofrecer una descripción teóricopráctica de los aspectos más relevantes de cada sesión, que puede ser descargada y visualizada por los alumnos tanto en la preparación previa de las sesiones, como durante ellas o incluso después, durante la preparación de los informes de resultados. El único aspecto que queda por cubrir para conseguir una integración adecuada de dichos recursos en el desarrollo de las sesiones es facilitar su accesibilidad, particularmente durante el desarrollo de las sesiones, que es cuando es más relevante la optimización del tiempo debido a su duración limitada. En este sentido, la realidad aumentada, que permite la combinación en tiempo real de la información digital y física usando dispositivos comunes como tabletas o teléfonos inteligentes, cobra especial relevancia, y en particular su realización como códigos QR.

Un código QR, término que proviene de las siglas en inglés de quick response (respuesta rápida), es un sistema de almacenamiento de información ideado para proporcionarla de manera inmediata. Los códigos QR se crearon en 1994 por Denso Wave para gestionar y controlar su inventario. Son una evolución de los códigos de barras tradicionales que toman la forma de matrices bidimensionales, lo que les permite el almacenamiento de una cantidad de información mucho mayor (7089 caracteres numéricos y 4296 alfanuméricos). Estas etiquetas, que son de código abierto y libres de licencias, se han utilizado masivamente desde sus primeras versiones a los

Octubre 4-6, 2017, Zaragoza, ESPAÑA

IV Congreso Internacional sobre Aprendizaje, Innovación y Competitividad (CINAIC 2017) 


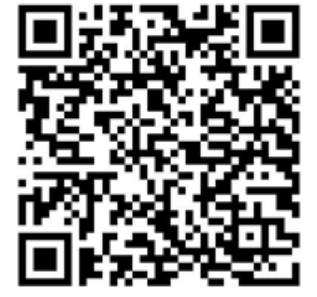

(a)

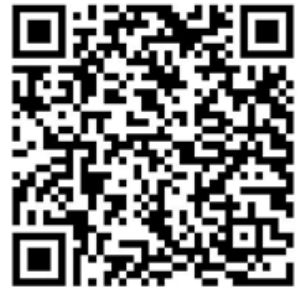

(b)
Fig. 2. Códigos QR generados para enlazar a (a) el manual del osciloscopio Agilent InfiniiVision 2000X y (b) un video mostrando cómo se mide el slew rate de un amplificador.

más recientes códigos iQR, que pueden ser generados tanto en formato cuadrado como rectangular. Los códigos más grandes tienen 422x422 módulos, lo que permite almacenar más de 40000 caracteres numéricos (Denso, 2009).

La información almacenada en un código QR puede ser un texto plano, un enlace a una URL, una imagen e incluso un vídeo, o también puede utilizarse para desencadenar acciones en los dispositivos tales como realizar una llamada telefónica, enviar un SMS o una tarjeta digital (vCard), mandar un correo electrónico, enlazar a un perfil en una red social, o añadir un evento a un calendario. Por estas razones, y sobre todo por el uso masivo de teléfonos inteligentes, los códigos QR se usan en un enorme número de actividades.

En Internet es posible encontrar infinidad de páginas dedicadas a la creación gratuita de códigos QR de manera rápida y sencilla. En otras, se pueden mencionar los generadores web Kaywa (http://qrcode.kaywa.com), UQR (https://uqr.me/), GoQR (http://goqr.me), o QRCode (http://qrcode.es), un sencillo generador que funciona sin necesidad de instalación o registro.

La lectura de la información almacenada en dichos códigos se realiza mediante dispositivos móviles, donde también es posible encontrar una gran variedad de aplicaciones gratuitas que realizan esta función, en todas las plataformas móviles. Algunas de ellas son QuickMark, ZXing Decoder Online o ScanLife.

El uso de códigos QR en educación permite establecer

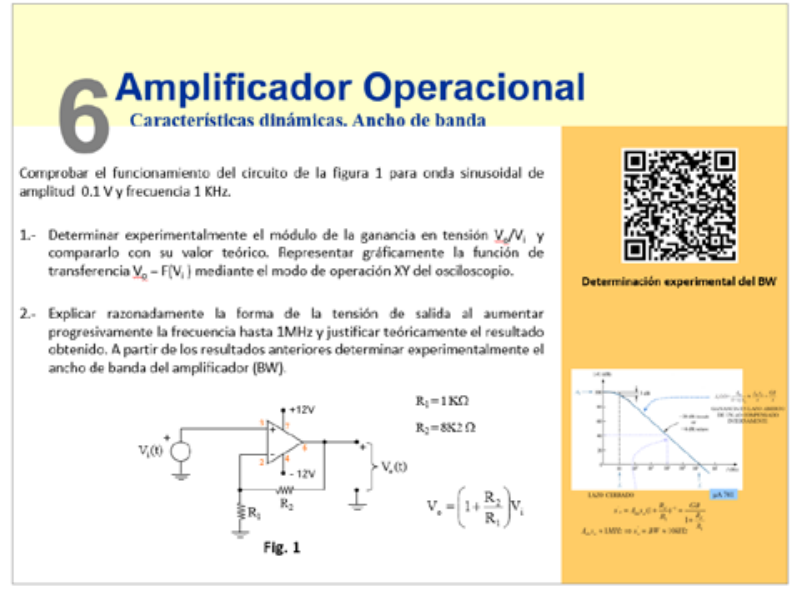

(a) enlaces entre elementos que se encuentran en el entorno educativo (notas, reportes, manuales...) e información accesible a través de Internet como textos con material de refuerzo, colecciones de problemas y ejercicios, fórums de discusión, páginas web con recursos específicos o demostraciones prácticas de ciertos procesos, por citar tan sólo unos cuantos.

\section{DESCRIPCIÓN}

En el desarrollo de este trabajo se ha generado una serie de contenidos multimedia orientados a las sesiones de prácticas que se llevan a cabo en un laboratorio de Electrónica. Dichos contenidos se enlazan desde el propio material presente en la sesión mediante códigos QR, en forma de etiquetas en los propios instrumentos o enlaces incrustados en los guiones de prácticas en un lugar relevante en el flujo de su desarrollo. Dichos recursos tienen como objetivo redundar en una mejora en el aprovechamiento del tiempo dedicado al laboratorio y en un aprendizaje más significativo.

Se han desarrollados dos tipos de recursos multimedia, asociados a sus propios códigos QR. Unos, llamados de Tipo 1 , se encuentran directamente en la instrumentación usada en el desarrollo de la práctica, y contienen hipervínculos a manuales, hojas de especificaciones, etc.; su objetivo es facilitar la consulta de información referente a la operación y el funcionamiento de la instrumentación requerida en el laboratorio (Fig. 1a). La segunda clase de códigos, llamados de Tipo 2, aparecen en la documentación que cada estudiante debe descargar para la realización de cada práctica (Fig. 1b) y, a través de ellos, los estudiantes tienen acceso a presentaciones comentadas de los aspectos teóricos y prácticos más relevantes, así como a vídeos con demostraciones prácticas de cómo es el proceso de medida de determinados parámetros en las fases más complejas del desarrollo de la práctica. Esto permite optimizar el uso del tiempo en el laboratorio, minimizando las dudas que se generan cuando se utiliza determinada instrumentación, mejorando las competencias en su manejo y permitiendo a los estudiantes concentrarse en el análisis de los resultados y en su relación con los contenidos teóricos.

La Fig. 2 muestra dos ejemplos de códigos QR generados

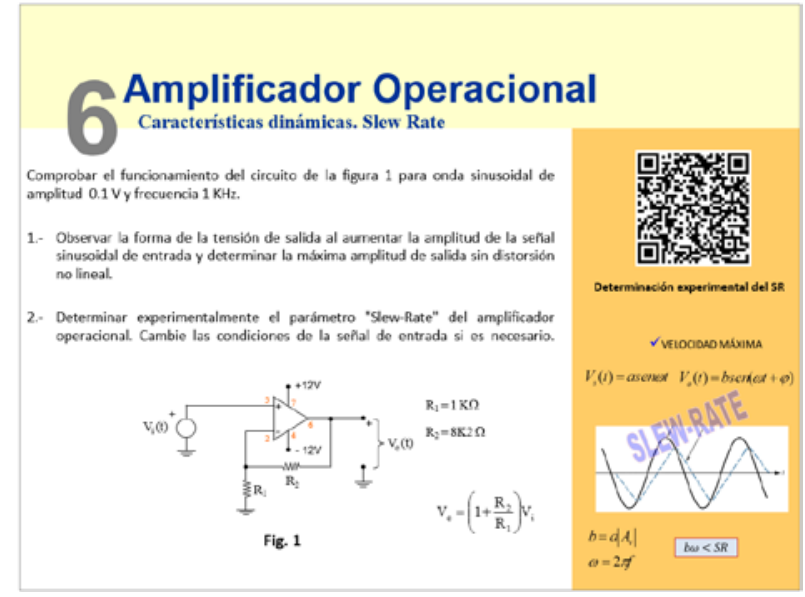

(b)

Fig 3. Detalle de la presentación de la sesión de laboratorio correspondiente al amplificador operacional. Explicación del proceso de medida y código QR que enlaza al vídeo demostrativo de (a) ancho de banda y (b) slew-rate del amplificador. 


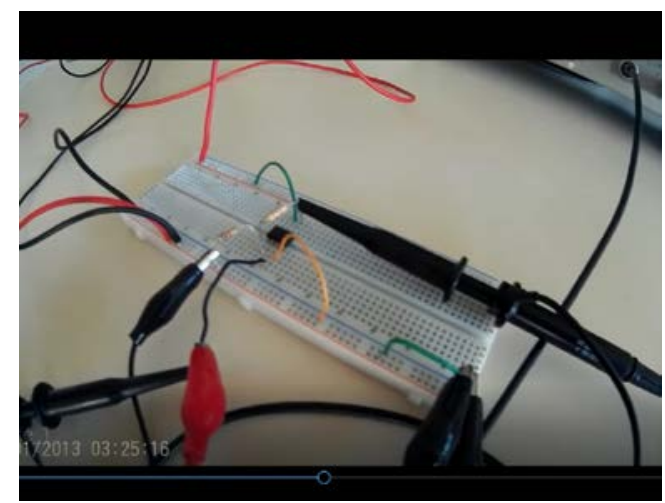

(a)

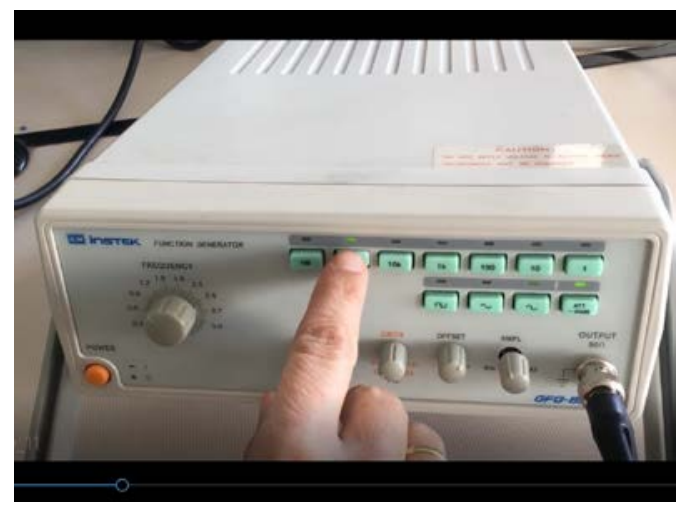

(b)

Fig 4. Ilustración mediante fotogramas de los vídeos editados para la explicación de las sesiones prácticas. En concreto, se aprecia el detalle de (a) la implementación del circuito y (b) el manejo de la instrumentación.

para esta experiencia, cada uno de los cuales pertenece a una de las dos categorías definidas: el código QR de la Fig. 2a proporciona un enlace al manual de usuario de un osciloscopio (Agilent InfiniiVision 2000X), mientras que el código QR de la Fig. 2b proporciona un enlace a un video tutorial que detalla el proceso de medida del ancho de banda de un amplificador no inversor usando el amplificador operacional de propósito general OA741.

Con el objetivo de que este recurso educativo tenga un carácter transversal a distintas asignaturas de grados científicos y tecnológicos, el primer tipo de códigos QR está orientado a elementos básicos de instrumentación tales como osciloscopios, generadores de señales y multímetros. Los códigos generados para incorporar los tutoriales se han orientado a detallar procesos comunes de medida en el ámbito de la Electrónica, como pueden ser las principales características de una señal, el ancho de banda de un sistema, el slew-rate de un amplificador operacional de propósito general o la ganancia en tensión de una etapa amplificadora, todos ellos conceptos generales presentes en las diversas asignaturas relacionadas con los circuitos y los dispositivos electrónicos. La Fig. 3 muestra ejemplo de códigos QR usados en la presentación explicativa de la sesión práctica.

Los códigos QR de Tipo 1 se generan para ser usados en el laboratorio desde el primer día. Por su parte, los códigos de Tipo 2 se han ido incorporando a la documentación en las fechas en las que son necesarias para la correcta realización de la práctica. Todos los contenidos que se han desarrollado para ser enlazados mediante códigos QR (vídeo tutoriales, manuales, información complementaria...) se han alojado en un curso permanente en la plataforma virtual de la institución. La Fig. 4 muestra dos fotogramas de los vídeos editados a tal fin.

\section{Resultados}

Además de los beneficios asociados con el m-learning, tales como acceso inmediato, interacción y personalización, e individualización del aprendizaje, podemos destacar que se ha conseguido una mejora en la autonomía de los estudiantes en la adquisición de competencias experimentales, ya que disponen en todo momento de un recurso activo de ayuda, particularizado al instrumento y al proceso de medida utilizado.

El objeto de aprendizaje propuesto en este trabajo, códigos QR que enlazan a contenidos multimedia generados específicamente para redundar en una mejora en el aprovechamiento del tiempo dedicado al laboratorio y en un aprendizaje más significativo, debe ser evaluado y desde una perspectiva múltiple, que abarque tanto los aspectos pedagógicos, tecnológicos o de interacción con la herramienta.

Basándonos en las seis características básicas que el estándar internacional para la evaluación de la calidad de productos software ISO 9126 establece como criterios de calidad, hemos seleccionado aquellas dimensiones que se ajustan mejor a nuestro recurso dimensionándolas adecuadamente y definimiento las preguntas correspondientes en cada dimensión. De esta manera, se ha elaborado un test para tal fin, cuyo extracto puede observarse en la Fig. 5. En líneas generales, podemos indicar que las preguntas más relevantes planteadas tienen por objetivo conocer si:

- $\quad$ El acceso al contenido multimedia enlazado es rápido, fiable y consistente

- $\quad$ El contenido multimedia enlazado es compatible con su visionado tanto en teléfonos inteligentes como tabletas

- $\quad$ El contenido multimedia enlazado es conciso, fáciles de seguir y está claramente explicado

Entre las estrategias diseñadas para evaluar cómo la acción implementada, la generación de un conjunto de contenidos multimedia aplicados a un laboratorio de Electrónica y su accesibilidad mediante códigos QR, mejora el proceso de aprendizaje en el laboratorio, podemos destacar una comparativa entre el tiempo invertido en realizar las sesiones prácticas con ayuda del material enlazado mediante los códigos QR y el registro que se tiene de ediciones pasadas, en las que no se disponía de dichos contenidos multimedia.

Por otro lado, existe una fuerte correlación entre la motivación y satisfacción de los estudiantes y el éxito de las actividades de aprendizaje virtual, ya que se asocian a un mayor aprovechamiento de los estudiantes (Regueras, 2009). En este sentido, se ha diseñado una encuesta específica para obtener información acerca de los resultados de la actividad 


\begin{tabular}{|c|}
\hline FUNCIONALIDAD $(25 \%)$ \\
\hline 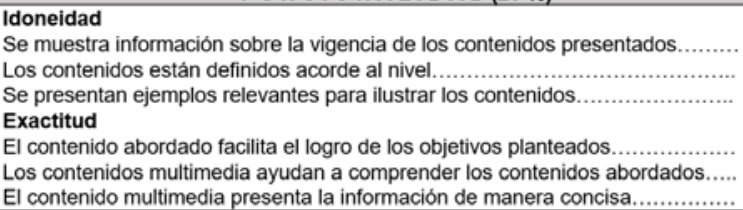 \\
\hline 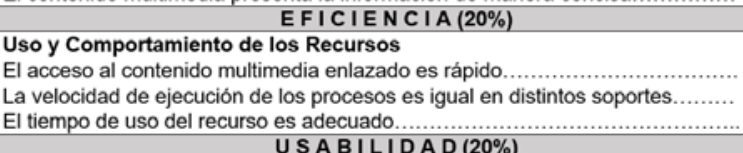 \\
\hline 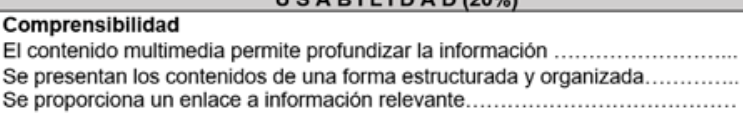 \\
\hline 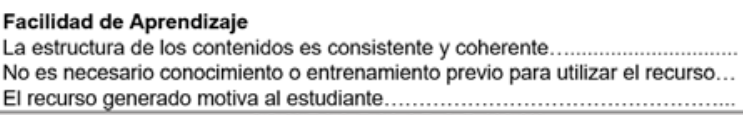 \\
\hline CONFIABILIDAD $(10 \%)$ \\
\hline $\begin{aligned} \text { En caso de error se puede continuar } & \text { M A N T E N I B I L I D A D (5\%) }\end{aligned}$ \\
\hline 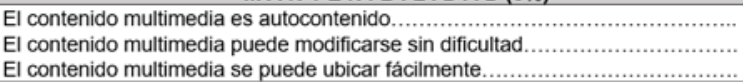 \\
\hline PORTABILIDAD $(20 \%)$ \\
\hline 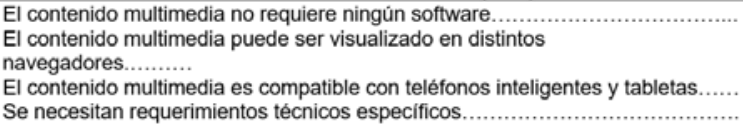 \\
\hline
\end{tabular}

Fig. 5. Ejemplo del test elaborado para evaluar la calidad del software según el estándar ISO 9126.

propuesta, en la que se presenta a los estudiantes una serie de afirmaciones antes las cuales deben indicar su grado de acuerdo siguiendo una escala de Likert. De entre los aspectos de interés del uso de los nuevos contenidos multimedia enlazados mediante códigos QR que se investigan en la encuesta, se ha preguntado si:

- $\quad$ El recurso proporciona enlaces a información relevante

- $\quad$ El recurso facilita la consulta de los manuales

- $\quad$ El recurso facilita el proceso de medida

- El contenido multimedia enlazado produce un mejor aprovechamiento del tiempo en el laboratorio

- $\quad$ El recurso minimiza el tiempo que empleo en buscar información

- El contenido multimedia enlazado mejora los resultados en el laboratorio

Estas medidas permitirán obtener, a juicio de los autores, una imagen fidedigna del impacto de la actividad sobre el proceso de aprendizaje de los estudiantes. Debido al estado actual de realización de la acción, se está poniendo en práctica este curso académico, no se dispone todavía de resultados cuantitativos.

\section{CONCLUSIONES}

En este trabajo se presenta el uso de códigos QR en un laboratorio de Electrónica para ofrecer información adicional y contextualizada que podrá ser consultada por los estudiantes en el momento en que lo necesiten. Con esta finalidad se han desarrollado dos tipos de códigos QR: los códigos de Tipo 1 se encuentran directamente sobre los instrumentos y ofrecen hipervínculos a manuales, hojas de especificaciones y otra información genérica, lo que facilita la consulta de información relativa a la operación de dichos instrumentos. Por su parte, los códigos de Tipo 2 se encuentran en la documentación de cada práctica, y proporcionan acceso a demostraciones prácticas del proceso de medida de determinados parámetros en las fases más complejas del desarrollo de cada una de las prácticas.

Mediante la implementación de esta experiencia se espera que los estudiantes se beneficien de una optimización del tiempo de realización de las sesiones prácticas, y también de una mayor profundidad en el análisis de los resultados y de su relación con los conceptos teóricos de la asignatura, consiguiendo así un aprendizaje más significativo. En particular en cursos en los que la relación entre el número de alumnos y profesores es alta, y por tanto en los que es complicado ofrecer atención inmediata a las dudas que van surgiendo en la realización de la sesión, el beneficio esperado será mayor.

Finalmente, se puede destacar que, pese a que la experiencia se presenta particularizada al ámbito de trabajo de los autores, puede extenderse de manera sencilla con el fin de mejorar el proceso de aprendizaje de competencias de tipo experimental en otras disciplinas científicas o técnicas.

\section{AgRADECIMIENTOS}

Este trabajo ha sido desarrollado en el marco de un Proyecto de Innovación Docente de la Universidad de Zaragoza (PIIDUZ_16_005).

\section{REFERENCIAS}

Cabero, J., Barroso, J. (2016). Posibilidades educativas de la realidad aumentada, New Approaches in Educational Research, 5(1), 46-52.

Cheon, J. (2012). An investigation of mobile learning readiness in higher education based on the theory of planned behavior, Computers \& Education, 59, 1054-1064.

Denso (2009). Recuperado de http://www.qrcode.com/en/index.html

Estebanell, M., Ferrés, J., Cornellà, P., Codina, D. (2012). Realidad aumentada y códigos QR en educación, Tendencias emergentes en educación con TIC. (pp. 277320). Barcelona: Editorial espiral.

Kossey, J., Berger, A. and Brown, V. (2015). Connecting to educational resources online with QR codes, FDLA Journal, 2, 1-10.

Law, C., So, S. (2010). QR codes in education, Journal of Educational Technology Development and Exchange, 3(1), 85-100.

Montero, Y. H. (2006). Factores del diseño web orientado a la satisfacción y no-frustración de uso, Revista Española de Documentación Científica, 29(2), 239-257.

Motiwalla, F. M. (2007). Mobile learning: A framework and evaluation, Computers \& Education, 49, 581-596.

Prendes C. (2015) Realidad aumentada y educación: análisis de experiencias prácticas, Revista de Medios y Educación, 46, 187-203.

Pu, H., Lin, J., Song, Y., Liu, F. (2011). Adaptive device context based mobile learning systems, International 
Journal of Distance Education Technologies (IJDET), 9(1), 44-56.

Regueras, L. M., Verdú, E., Muñoz, M. F., Pérez, M. A., de Castro, J. P., Verdú, M.J., (2009). Effects of competitive elearning tools on higher education students: A case study, IEEE Transactions on Education, 52(2), 279-285.

Sánchez-Azqueta, C., Gimeno, C., Celma, S., Aldea, C., (2015-1). E-learning environment for Electronics in Physics Degree, International Symposium on Project Approaches in Engineering Education (PAEE), 127-134.
Sánchez-Azqueta, C., Gimeno, C., Celma, S., Aldea, C., (2015-2). Using the Wiimote to learn MEMS in a Physics degree program, IEEE Transactions on Education, 59(3), 169-174.

Sánchez-Azqueta, C., Gimeno, C., Celma, S., Aldea, C., (2016). Enhanced eBooks in the teaching/learning process of electronics, $2^{\text {nd }}$ International Conference on Higher Education Advances (HEAD), 84-91. 